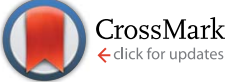

Cite this: J. Mater. Chem. A, 2016, 4, 3249

Received 12th January 2016 Accepted 2nd February 2016

DOI: 10.1039/c6ta00320f

www.rsc.org/MaterialsA

\section{Dynamic switching of ionic conductivity by cooperative interaction of polyviologen and liquid crystals for efficient charge storage $\uparrow$}

\author{
Kan Sato, Takaaki Yamasaki, Takahiro Mizuma, Kenichi Oyaizu* and Hiroyuki Nishide*
}

\begin{abstract}
The ionic conductivity of a liquid crystal electrolyte was switched along with redox reactions of polyviologen. The surface charge of the polymer determined the alignment and the bulk conductivity of the electrolyte. The cooperative switching firstly enabled the suppression of self-discharging and quick-response, giving rise to facile and persistent charge storage.
\end{abstract}

Organic redox-active polymers have attracted substantial attention as charge storage materials for secondary batteries, electrochromic devices, sensors, and memories. ${ }^{1-7}$ The devices exhibit fast charging/discharging performances in lightweight and flexible or even stretchable forms due to the chemical and mechanical robustness of the organic polymers. ${ }^{8-11}$ In order to improve battery performances, a series of studies with different concepts of polymer design were reported. ${ }^{12-17}$ The use of unconjugated polymer backbones enabled successful charging/ discharging with theoretical capacities whilst conjugated ones typically tended to suffer from chemical instability along with the high ratio of doping. ${ }^{18,19}$ Cross-linking structures suppressed the elution of polymers into electrolytes which may cause the loss of the capacity and even self-discharging induced by the shuttle process. ${ }^{20,21}$ Multi-electron redox-active moieties such as anthraquinones increased the theoretical capacity as electrodes, ${ }^{22,23}$ and composite electrodes with optimized compositions of redox-polymers and conductive carbons were also essential to maintain both power and energy density. ${ }^{24}$

Apart from electrode-active materials, emerging functional electrolytes such as ionic liquids have also attracted considerable attention for further favourable performances. ${ }^{25,26}$ Appropriate combinations of ionic species as electrolyte layers enabled the desirable properties such as ionic conductivity, viscosity, potential window, vapour pressure, and other important functions. ${ }^{25,27}$ In particular, liquid crystal electrolytes

Department of Applied Chemistry, Waseda University, Tokyo 169-8555, Japan. E-mail: nishide@waseda.jp; Fax: +81-3-3209-5522; Tel: +81-3-3200-2669

$\dagger$ Electronic supplementary information (ESI) available. See DOI: $10.1039 / \mathrm{c} 6 \mathrm{ta00320f}$ containing ionic liquids have been reported as a new class of functional electrolytes, owing to their anisotropic ionic

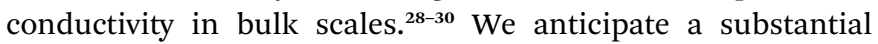
potential of using them for charge storage applications because their ionic conductivity can be, in theory, reversibly tuned in favoured directions ${ }^{28,31}$ along with charge/discharge reactions, and it will contribute to both the quick response of the devices and the suppression of unfavourable self-discharging.

Herein we report a new technique of dynamically switching the ionic conductivity of liquid crystal electrolytes by cooperatively controlling the redox reaction of polyviologen 1 (ref. 32) to induce the alignment of the liquid crystal electrolyte containing nematic liquid crystal 4-cyano-4'-pentylbiphenyl (5CB) and ionic liquid 1-ethyl-3-methylimidazolium tetracyanoborate (EMIM TCB) which exhibits the anisotropic ionic conductivity. The bulk ionic conductivity of the device was reversibly tuned along with the charge/discharge reactions by changing the alignment of the liquid crystals. High conductivity was achieved during the charging/discharging reactions, but low conductivity was obtained only after the completion of the charge (Fig. 1). The highly conductive state during the redox reaction should result in quick responses of the devices, and the lower conductivity that emerges only in the fully charged state should suppress the undesired self-discharging induced by the shuttle processes of the ionic species. ${ }^{33,34}$

We expected that the alignment of 5CB could be in principle controlled by adjusting the force balances of (a) the electric field of the double layer in the vicinity of the electrode and (b) the Van der Waals force between the polyviologen and 5CB. When polyviologen 1 is in the dication state, perpendicular alignment of 5CB should be induced because of the electrostatic interaction between the dipole moment of 5CB (dielectric anisotropy $\Delta \varepsilon=+13)^{31}$ and the diffusion layer's electric field originating from the densely populated charge on the polyelectrolyte. On the other hand, when polyviologen 1 was one-electron reduced with each redox moiety, the Van der Waals force between the $\mathbf{1}$ and 5CB would become dominant and then induce the planar alignment of liquid crystals. The surface driven switching of 


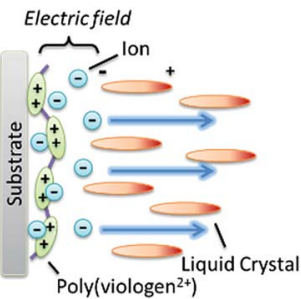

(a) Homeotropic alignment (High ionic conductivity)

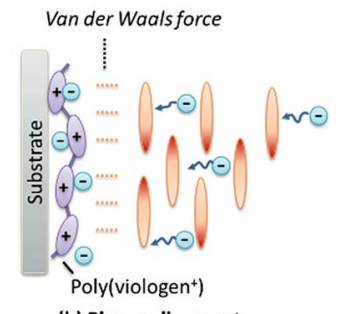

(b) Planar alignment (Low ionic conductivity)

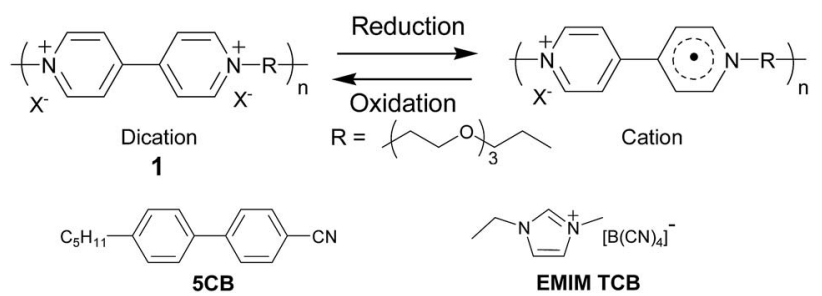

Fig. 1 Dynamic switching of ionic conductivity by cooperative interaction of polyviologen and nematic liquid crystals.

liquid crystals by tuning the balance of the electric double layer and Van der Waals force described above was preliminary studied by Abbot's group, but they examined only the cases and mechanisms of self-assembled monolayers of ferrocene or redox-inactive compounds for optical applications. ${ }^{31,35}$ Our present report will be the first to successfully use the redox polymers and the liquid crystal electrolytes for the dynamic tuning of ionic conductivity enabling the efficient charge storage.

The electrochromic device was fabricated using a spincoated 1 layer on an ITO substrate as the electrochromic electrode, ATO nanoparticles on an ITO as the capacitive counter, and $35 \mathrm{mM}$ EMIM TCB in 5CB as the electrolyte solution. We selected EMIM TCB because it exhibited higher tendency for homeotropic alignment compared to former reported common electrolytes including tetrabutylammonium tetrafluoroborate $\left(\mathrm{TBABF}_{4}\right)$ (Table S1, further discussion in the ESI $\left.\dagger\right){ }^{31}$ Hydrophobic ionic liquids exhibited more favourable miscibility and higher degrees of dissociation in 5CB which should contribute to the strong electric field of the double layer in the vicinity of the charged polymer and induce homeotropic alignment (Fig. S1 and S2 $\dagger$ ). The reversible electrochromic response was observed by applying constant voltages to the device (Fig. 2).

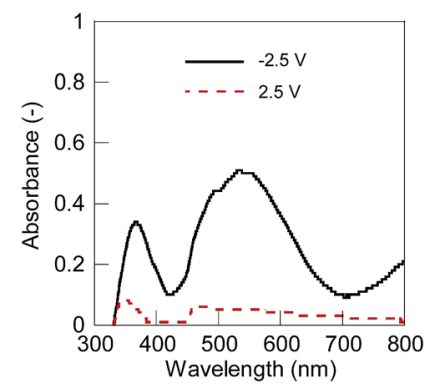

Fig. 2 UV-vis spectra of the electrochromic device. Solid line (black): $-2.5 \mathrm{~V}$, and dashed line (red): $2.5 \mathrm{~V}$.
The polymer layer in the discharged dicationic state was transparent at 0 and $2.5 \mathrm{~V} v$ s. the capacitor electrode but turned to red at $-2.5 \mathrm{~V}$ by reduction into the charged cationic state. The results suggested the reversible redox reactions of $\mathbf{1}$ between the dication and cation states. Simultaneously, we observed the dynamic alignment changes of 5CB accompanied with the redox reactions (Fig. 3). Polarized light microscopy observation showed the homeotropic alignment in the dication state and planar or tilted alignment in the cation state. In the former state, the strongly charged surfaces of both $\mathbf{1}$ and the metallic oxide (ATO, counter electrode) ${ }^{30}$ have certainly induced the strong electric field of the double layer at the surfaces, and caused homeotropic alignment. On the other hand, the planar or tilted alignment in the cation state should be induced by dominantly affecting the Van der Waals force between 1 and liquid crystals after the decrease in the polymer charge by the reduction of the dication to the cation. 5CB molecules close to the counter electrode seemed to always align perpendicularly against the substrate as expected in the absence of substantial redox reactions at the surface..$^{31}$

Dynamically switching the alignment of the liquid crystal along with the redox reactions enabled both quick charging/ discharging and suppression of self-discharging. For comparison, we used a $1000 \mathrm{mT}$ external magnetic field induced by neodymium magnets to always homeotropically align 5CB against substrates. ${ }^{36}$ We observed the significant difference in ionic conductivity between the planar $\left(8.25 \times 10^{-6} \mathrm{~S} \mathrm{~cm}\right.$ without magnet) and homeotropically aligned $\left(8.70 \times 10^{-6} \mathrm{~S} \mathrm{~cm}\right.$ with magnet) conditions after charging (Fig. 4(a)). The difference in the resistivity was calculated to be $6300 \Omega \mathrm{cm}$ and the ratio was 1.05 , which was slightly lower than the original anisotropic ratio of the electrolyte (1.13 in Fig. S3†) presumably because 5CB always aligned perpendicularly in the vicinity of the counter ATO electrode. The rate of self-discharging was successfully reduced under the planar alignment conditions compared to the homeotropic state (Fig. 4(b)). Open circuit potentials differed up to 200 seconds and the result suggested that the low ionic conduction of the electrolyte with the planar alignment efficiently suppressed the unfavourable self-discharging reactions with the ionic species.

After 200 seconds, the alignment of 5CB changed from planar to homeotropic, and no significant difference was observed between the conditions with or without the external magnetic field. The alignment change from homeotropic to
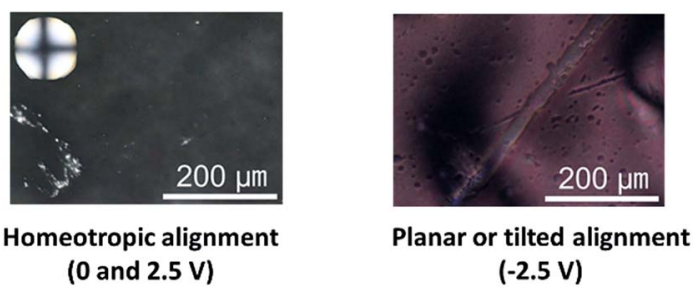

Fig. 3 Polarized light microscopy images of the electrochromic device. Inset: conoscope observation. The cross-shape indicates the homeotropic alignment of liquid crystals. 

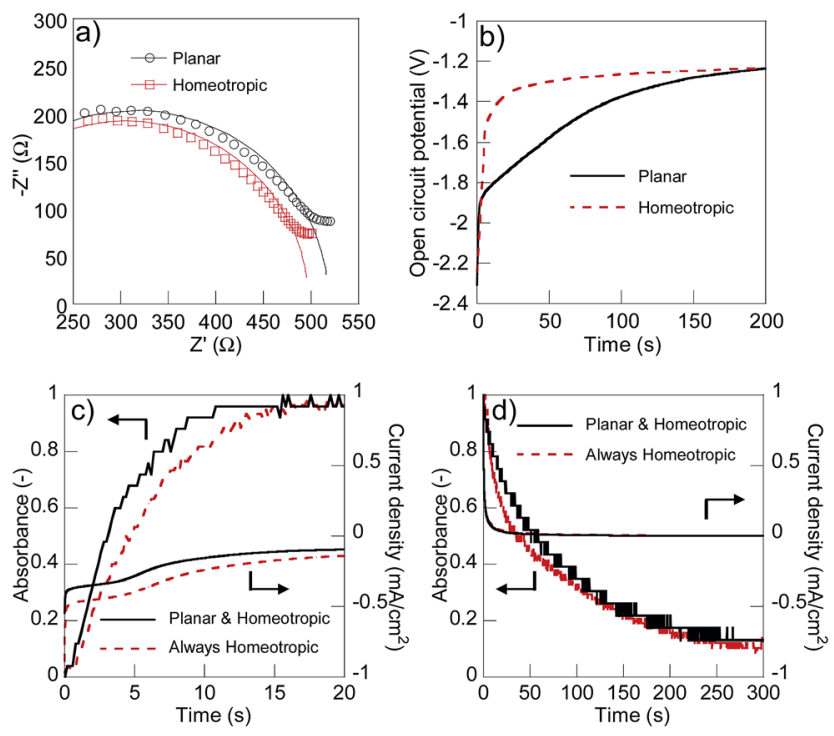

Fig. 4 (a) Electrochemical impedance spectra of the electrochromic device at $-2.5 \mathrm{~V}\left(10^{6}\right.$ to $5 \times 10^{4} \mathrm{~Hz}, \mathrm{AC}$ amplitude $\left.10 \mathrm{mV}\right)$. (b) Open circuit potential of the device after charging at $-2.5 \mathrm{~V}$. (c) Time dependences of the absorbance at $530 \mathrm{~nm}$ and current density applied at $-2.5 \mathrm{~V}$, and (d) $2.5 \mathrm{~V}$. The magnetic field $(1000 \mathrm{mT})$ was applied to induce the homeotropic alignment (dotted line).

planar should be caused by the self-discharging of the polymer surface. The use of liquid crystal electrolytes with even higher anisotropic ionic conductivity will more efficiently suppress the unfavourable self-discharging reactions. We observed no significant difference in the response rate for the charge/ discharge reactions between the dynamically changed alignment in the absence of the magnet and permanently homeotropically aligned conditions using the magnet (Fig. 4(c) and (d)). Both devices turned on in 15 seconds, and decoloured in 300 seconds. Since the alignment of 5CB was homeotropic except for the fully charged state, the high ionic conductivity during the charge/discharge processes seemed to enable the fast response rate as high as that of the permanently homeotropic conditions with the magnet. The slower responses for decolouring than colouring should be caused partially by the dense stacking of viologen cations. ${ }^{37}$ Enhancing the switching ratio of ionic conductivity with a view to accomplish more significant co-operation is the topic of our continuous research.

\section{Conclusions}

We established a new technique of dynamically switching ionic conductivity of electrolytes with a difference of $6300 \Omega \mathrm{cm}$ utilizing the cooperative interaction of redox polymers and liquid crystals. The favourable change of the ionic conductivity along with the charge/discharge reactions enabled both the significant suppression of the self-discharging caused by ionic species and quick response of the device in 15 seconds. To the best of our knowledge, this is the first report on dynamic tuning of the ionic conductivity of electrolytes along with the charging/ discharging by polymers which enables efficient charge storage.
Our technique can be widely used for a series of reported liquid crystal electrolytes with higher regularity and anisotropic ionic conductivity, ${ }^{28,38}$ and a further efficient charge storage system can be realized by using them. The successful alignment will lead to facile and persistent charge storage with potential applications to electrochromic devices and batteries with dramatically enhanced performances.

\section{Acknowledgements}

This work was supported by Grants-in-Aid for JSPS Fellows (No. 15J00888), Scientific Research (No. 24225003, 26620108, and 25288056), and the Leading Graduate Program in Science and Engineering, Waseda University from MEXT, Japan.

\section{Notes and references}

$1 \mathrm{H}$. Nishide and K. Oyaizu, Science, 2008, 319, 737-738.

2 Z. Song and H. Zhou, Energy Environ. Sci., 2013, 6, 2280.

3 S. V. Vasilyeva, P. M. Beaujuge, S. Wang, J. E. Babiarz, V. W. Ballarotto and J. R. Reynolds, ACS Appl. Mater. Interfaces, 2011, 3, 1022-1032.

4 Y. Yonekuta, K. Susuki, K. Oyaizu, K. Honda and H. Nishide, J. Am. Chem. Soc., 2007, 129, 14128-14129.

5 M. K. Park, S. Deng and R. C. Advincula, J. Am. Chem. Soc., 2004, 126, 13723-13731.

6 J. C. Forgie, A. L. Kanibolotsky, P. J. Skabara, S. J. Coles, M. B. Hursthouse, R. W. Harrington and W. Clegg, Macromolecules, 2009, 42, 2570-2580.

7 C. Hu, T. Sato, J. Zhang, S. Moriyama and M. Higuchi, ACS Appl. Mater. Interfaces, 2014, 6, 9118-9125.

8 H. Nishide, K. Koshika and K. Oyaizu, Pure Appl. Chem., 2009, 81, 1961-1970.

9 T. Suga, H. Ohshiro, S. Sugita, K. Oyaizu and H. Nishide, $A d v$. Mater., 2009, 21, 1627-1630.

10 T. Suga, S. Sugita, H. Ohshiro, K. Oyaizu and H. Nishide, Adv. Mater., 2011, 23, 751-754.

11 N. Sano, W. Tomita, S. Hara, C.-M. Min, J.-S. Lee, K. Oyaizu and H. Nishide, ACS Appl. Mater. Interfaces, 2013, 5, 13551361.

12 T. Janoschka, M. D. Hager and U. S. Schubert, Adv. Mater., 2012, 24, 6397-6409.

13 P. Novák, K. Müller, K. S. V. Santhanam and O. Haas, Chem. Rev., 1997, 97, 207-282.

14 I. S. Chae, M. Koyano, T. Sukegawa, K. Oyaizu and H. Nishide, J. Mater. Chem. A, 2013, 1, 9608-9611.

15 W. Choi, S. Endo, K. Oyaizu, H. Nishide and K. E. Geckeler, J. Mater. Chem. A, 2013, 1, 2999-3003.

16 K. Oyaizu, A. Hatemata, W. Choi and H. Nishide, J. Mater. Chem., 2010, 20, 5404-5410.

17 J. P. Blinco, J. L. Hodgson, B. J. Morrow, J. R. Walker, G. D. Will, M. L. Coote and S. E. Bottle, J. Org. Chem., 2008, 73, 6763-6771.

18 K. Oyaizu and H. Nishide, Adv. Mater., 2009, 21, 2339-2344. 19 J. C. W. Chien and J. B. Schlenoff, Nature, 1984, 311, 362363. 
20 J. K. Feng, Y. L. Cao, X. P. Ai and H. X. Yang, J. Power Sources, 2008, 177, 199-204.

21 K. Oyaizu, Y. Ando, H. Konishi and H. Nishide, J. Am. Chem. Soc., 2008, 130, 14459-14461.

22 B. Huskinson, M. P. Marshak, C. Suh, S. Er, M. R. Gerhardt, C. J. Galvin, X. Chen, A. Aspuru-Guzik, R. G. Gordon and M. J. Aziz, Nature, 2014, 505, 195-198.

23 W. Choi, D. Harada, K. Oyaizu and H. Nishide, J. Am. Chem. Soc., 2011, 133, 19839-19843.

24 T. Sukegawa, K. Sato, K. Oyaizu and H. Nishide, RSC Adv., 2015, 5, 15448-15452.

25 A. Lewandowski and A. Świderska-Mocek, J. Power Sources, 2009, 194, 601-609.

26 M. Armand, F. Endres, D. R. MacFarlane, H. Ohno and B. Scrosati, Nat. Mater., 2009, 8, 621-629.

27 M.-C. Lin, M. Gong, B. Lu, Y. Wu, D.-Y. Wang, M. Guan, M. Angell, C. Chen, J. Yang, B.-J. Hwang and H. Dai, Nature, 2015, 520, 324-328.

28 T. Kato, N. Mizoshita and K. Kishimoto, Angew. Chem., Int. Ed., 2005, 45, 38-68.
29 M. Yoshio, T. Mukai, K. Kanie, M. Yoshizawa, H. Ohno and T. Kato, Adv. Mater., 2002, 14, 351-354.

30 R. Rondla, J. C. Y. Lin, C. T. Yang and I. J. B. Lin, Langmuir, 2013, 29, 11779-11785.

31 Y.-Y. Luk and N. L. Abbott, Science, 2003, 301, 623-626.

32 R. H. Terrill, J. E. Hutchison and R. W. Murray, J. Phys. Chem. $B$, 1997, 101, 1535-1542.

33 A. Mirmohseni and R. Solhjo, Eur. Polym. J., 2003, 39, 219223.

34 N. Oyama, N. Oki, H. Ohno, Y. Ohnuki, H. Matsuda and E. Tsuchida, J. Phys. Chem., 1983, 87, 3642-3647.

35 R. R. Shah and N. L. Abbott, J. Phys. Chem. B, 2001, 105, 4936-4950.

36 Y. Xiang, Z. Lin, T. Li, X. Liang, J. Zhang, J. W. Huang and L. N. Ji, Jpn. J. Appl. Phys., Part 1, 1999, 38, 6017-6018.

37 T. M. Bockman and J. K. Kochi, J. Org. Chem., 1990, 55, 41274135.

38 J. Sakuda, E. Hosono, M. Yoshio, T. Ichikawa, T. Matsumoto, H. Ohno, H. Zhou and T. Kato, Adv. Funct. Mater., 2015, 25, 1206-1212. 